\title{
О некоторых функциональных свойствах трехзначных матриц для классической логики
}

\author{
Л. Ю.ДЕвяткин
}

\begin{abstract}
In this paper a number of functional properties of implicativenegative three-valued logical matrices with the classical cosequence relation is described.

Ключевые слова: классическая пропозициональная логика, трехзначная логика, отношение логического следования, логические матрицы
\end{abstract}

В данной статье будет рассмотрен ряд трехзначных семантик для классической пропозициональной логики, построенных с помощью логических матриц.

Дадим ряд необходимых определений.

ОПРЕДЕЛЕНИЕ 1 (Алфавит пропозиционального языка $L_{\supset}$ ). Алфавиту пропозиционального языка $L_{\supset}$ принадлежат только следующие символы: бинарная логическая связка つ, унарная логическая связка ᄀ, пропозициональные переменные $p_{1}, p_{2}$, $p_{3}, \ldots$, а также скобки.

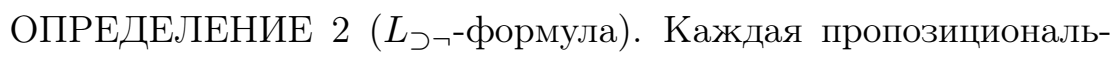

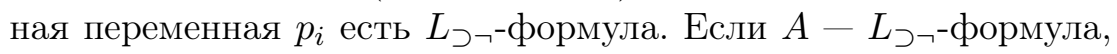

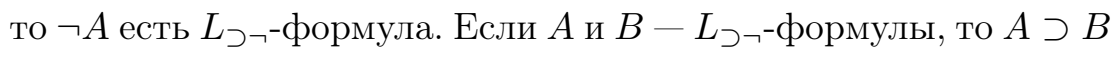

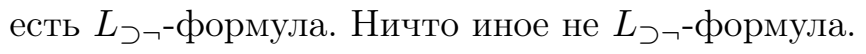

ОПРЕДЕЛЕНИЕ 3 (Логическая матрица). Будем называть логической матрицей $M=\langle U, F, D\rangle$, где $U-$ непустое множество, $D$ - непустое подмножество $U$, интерпретируемое как множество выделенных значений, $F$ - множество операций, заданных на $U$. 
Если не указано иное, будем рассматривать матрицы, в которых множество $F$ содержит в точности одну бинарную и одну унарную базовые операции.

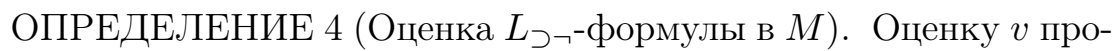

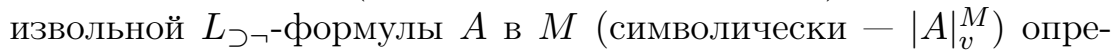
делим обычным образом: $|p|_{v}^{M} \in U$, если $p$ есть пропозицио-

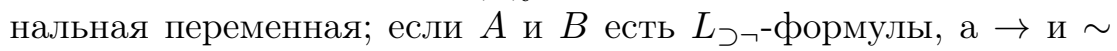
есть соответственно бинарная и унарная базовые операции $M$, то $|A \supset B|_{v}^{M}=|A|_{v}^{M} \rightarrow|B|_{v}^{M},|\neg A|_{v}^{M}=\sim|A|_{v}^{M}$.

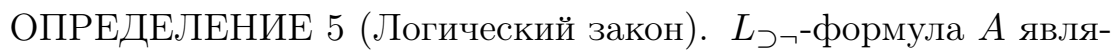
ется законом в логической матрице $M$, е.т.е. $|A|_{v}^{M} \in D$ при каждой оценке $v$ в $M$.

ОПРЕДЕЛЕНИЕ 6 (Отношение логического следования). $L_{\supset \neg}$ формула $B$ логически следует из множества посылок $\Gamma$ в $M$ (символически $\Gamma \models_{M} B$ ), е.т.е. не существует оценки $v$ в $M$, при которой все формулы из $\Gamma$ принимают выделенное значение, а формула $B$ принимает невыделенное значение.

ОПРЕДЕЛЕНИЕ 7 (Классическое отношение логического следования в $M)$. Пусть логическая матрица $M_{2}=\left\langle\{1,0\}, \supset^{+}, \neg^{+}\right.$, $\{1\}\rangle$ есть матрица для классической пропозициональной логики, a $\supset^{+}$и $\neg^{+}$определяются стандартными таблицами истинности для импликации и отрицания. Пусть $M_{3}=\left\langle\left\{1, \frac{1}{2}, 0\right\}, \supset^{*}, \neg^{*},\{1\}\right\rangle$ есть произвольная матрица с трехэлементным множеством-носителем, на котором заданы одна бинарная и одна унарная базовые операции. Будем говорить, что отношение логического следования в $M_{3}$ является классическим, если и только если выполняется следующе условие: $\Gamma \models_{M_{2}} B$, е.т.е. $\Gamma \models_{M_{3}} B$.

Можно доказать следующую теорему [2]:

TEOРЕМА 1. Отношение логического следования в $M_{3}$ является классическим, только когда базовые связки $M_{3}$ отвечают следующим условиям: $x \supset^{*} y=1$, е.т.е. $x \in\left\{\frac{1}{2}, 0\right\}$ или $y=1$, в противном случае $x \supset^{*} y \in\left\{\frac{1}{2}, 0\right\} ; \neg^{*} x=1$, e.m.e. $x \in\left\{\frac{1}{2}, 0\right\}$, в противном случае $\neg^{*} x \in\left\{\frac{1}{2}, 0\right\}$.

Существует восемь наборов связок, отвечающих данному условию: 


\begin{tabular}{|c|ccc|}
\hline$\supset^{\alpha}$ & 1 & $\frac{1}{2}$ & 0 \\
\hline 1 & 1 & 0 & 0 \\
$\frac{1}{2}$ & 1 & 1 & 1 \\
0 & 1 & 1 & 1 \\
\hline
\end{tabular}

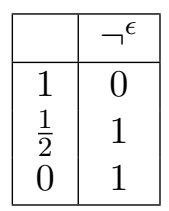

\begin{tabular}{|c|ccc|}
\hline$\supset^{\beta}$ & 1 & $\frac{1}{2}$ & 0 \\
\hline 1 & 1 & $\frac{1}{2}$ & 0 \\
$\frac{1}{2}$ & 1 & 1 & 1 \\
0 & 1 & 1 & 1 \\
\hline
\end{tabular}

\begin{tabular}{|c|c|}
\hline & $\neg^{\epsilon}$ \\
\hline 1 & 0 \\
$\frac{1}{2}$ & 1 \\
0 & 1 \\
\hline
\end{tabular}

\begin{tabular}{|c|ccc|}
\hline$\supset^{\gamma}$ & 1 & $\frac{1}{2}$ & 0 \\
\hline 1 & 1 & 0 & $\frac{1}{2}$ \\
$\frac{1}{2}$ & 1 & 1 & 1 \\
0 & 1 & 1 & 1 \\
\hline
\end{tabular}

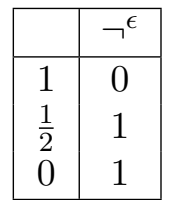

\begin{tabular}{|c|ccc|}
\hline$\supset^{\delta}$ & 1 & $\frac{1}{2}$ & 0 \\
\hline 1 & 1 & $\frac{1}{2}$ & $\frac{1}{2}$ \\
$\frac{1}{2}$ & 1 & 1 & 1 \\
0 & 1 & 1 & 1 \\
\hline
\end{tabular}

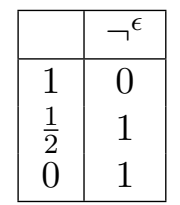

\begin{tabular}{|c|ccc|}
\hline$\supset^{\alpha}$ & 1 & $\frac{1}{2}$ & 0 \\
\hline 1 & 1 & 0 & 0 \\
$\frac{1}{2}$ & 1 & 1 & 1 \\
0 & 1 & 1 & 1 \\
\hline
\end{tabular}

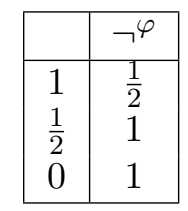

\begin{tabular}{|c|ccc|}
\hline$\supset^{\beta}$ & 1 & $\frac{1}{2}$ & 0 \\
\hline 1 & 1 & $\frac{1}{2}$ & 0 \\
$\frac{1}{2}$ & 1 & 1 & 1 \\
0 & 1 & 1 & 1 \\
\hline
\end{tabular}

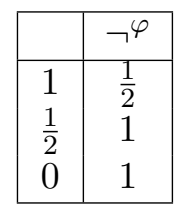

\begin{tabular}{|c|ccc|}
\hline$\supset^{\gamma}$ & 1 & $\frac{1}{2}$ & 0 \\
\hline 1 & 1 & 0 & $\frac{1}{2}$ \\
$\frac{1}{2}$ & 1 & 1 & 1 \\
0 & 1 & 1 & 1 \\
\hline
\end{tabular}

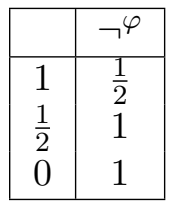

\begin{tabular}{|c|ccc|}
\hline$\supset^{\delta}$ & 1 & $\frac{1}{2}$ & 0 \\
\hline 1 & 1 & $\frac{1}{2}$ & $\frac{1}{2}$ \\
$\frac{1}{2}$ & 1 & 1 & 1 \\
0 & 1 & 1 & 1 \\
\hline
\end{tabular}

\begin{tabular}{|c|c|}
\hline & $\neg^{\varphi}$ \\
\hline 1 & $\frac{1}{2}$ \\
$\frac{1}{2}$ & 1 \\
0 & 1 \\
\hline
\end{tabular}

Таким образом, может быть построено восемь логических матриц с трехэлементным множеством-носителем, в которых отношение логического следования является классическим. Обозначим их соответственно как $M_{3}^{\alpha, \epsilon}, M_{3}^{\beta, \epsilon}, M_{3}^{\gamma, \epsilon}, M_{3}^{\delta, \epsilon}, M_{3}^{\alpha, \varphi}, M_{3}^{\beta, \varphi}$, $M_{3}^{\gamma, \varphi}, M_{3}^{\delta, \varphi}$. Некоторые из перечисленных связок достаточно известны. Так, первый набор связок - это внешние импликаци и отрицание трехзначной логики Бочвара $\mathbf{B}_{\mathbf{3}}$ [1]. Связка $\supset^{\beta}$ была независимо описана в целом ряде работ $[4,5,6]$.

Как известно (см., например, [3]), классические импликация и отрицание образуют функционально полную систему связок, т. е. с их помощью может быть выражена любая функция, заданная на $\{1,0\}$. Интересно, что в случае с приведенными выше наборами связок ситуация совершенно иная.

УТВЕРЖДЕНИЕ 1. Используя базовые операции $M_{3}^{\alpha, \epsilon} u M_{3}^{\delta, \varphi}$, нельзя выразить никакие иные из описанных нами связок.

Доказательство. Ясно, что любая функция, выразимая в данных матрицах, имеет область значения $\{1,0\}$ или $\left\{1, \frac{1}{2}\right\}$ соответственно. Однако это неверно для остальных матриц.

Q.E.D. 
УТВЕРЖДЕНИЕ 2. В $M_{3}^{\beta, \epsilon}$ выразимы связки $M_{3}^{\alpha, \epsilon}$ и не выразимы базовые связки остальных матрии.

Доказательство. Импликация из $M_{3}^{\alpha, \epsilon}$ выражается следующим образом: $x \supset^{\alpha} y=\neg^{\epsilon} \neg^{\epsilon}\left(x \supset^{\beta} y\right)$. В то же время все функции $M_{3}^{\beta, \epsilon}$ имеют область значений $\{1,0\}$ при ограничении значений переменных тем же множеством. Однако это неверно для остальных матриц.

Q.E.D.

УТВЕРЖДДНИЕ 3. В $M_{3}^{\beta, \varphi}$ выразимы связки $M_{3}^{\delta, \varphi}$ и не выразимы базовые связки остальных матрии.

Доказательство. Импликация из $M_{3}^{\delta, \varphi}$ выражается аналогично предыдущему случаю: $x \supset^{\delta} y=\neg^{\varphi} \neg^{\varphi}\left(x \supset^{\beta} y\right)$.

Покажем, что через базовые операции $M_{3}^{\beta, \varphi}$ нельзя выразить унарный оператор $f^{1}$, такой, что $f^{1}(1)=0$.

Индуктивное допущение. Пусть $f^{1}$ нельзя выразить в $M_{3}^{\beta, \varphi}$, используя менее $k$ вхождений $\supset^{\beta}$ и $\neg^{\varphi}$.

Теперь допустим, что $f^{1}$ можно выразить посредством суперпозиции $g$ операций $\supset^{\beta}$ и $\neg^{\varphi}$, содержащей в точности $k$ вхождений данных операций.

Случай $1 . g(x)=\neg^{\varphi} h(x)$. Тогда $\neg^{\varphi} h(1)=0$. Однако это невозможно в силу определения $\neg^{\varphi}$.

Случай 2. $g(x)=h^{\prime}(x) \supset^{\beta} h^{\prime \prime}(x)$.

1. $h^{\prime}(1) \supset^{\beta} h^{\prime \prime}(1)=0$ (по условию)

2. $h^{\prime}(1)=1$ и $h^{\prime \prime}(1)=0$ (по определению $\supset^{\beta}$ )

3. $h^{\prime \prime}(1)=0$. Число вхождений $\supset^{\beta}$ и $\neg^{\varphi}$ в $h^{\prime \prime}$ меньше $k$. противоречие с индуктивным допущением.

Индукция закончена. Оператор $f^{1}$ невыразим в $M_{3}^{\beta, \varphi}$.

Однако этот оператор выразим в остальных матрицах: $x \supset^{\alpha}$ $\neg^{\varphi} x$ для $M_{3}^{\alpha, \varphi}, x \supset^{\gamma} \neg^{\varphi} x$ для $M_{3}^{\gamma, \varphi}, \neg^{\epsilon} x$ для остальных матриц.

Q.E.D.

УТВЕРЖДЕНИЕ 4. $M_{3}^{\delta, \epsilon}$ функционально эквивалентна $M_{3}^{\alpha, \varphi}$. В этих матрицах выразимы базовые операции $M_{3}^{\alpha, \epsilon}$ u $M_{3}^{\delta, \varphi} u$ не выразимы базовые операчии $M_{3}^{\beta, \epsilon}, M_{3}^{\beta, \varphi}, M_{3}^{\gamma, \epsilon} u M_{3}^{\gamma, \varphi}$. 
Доказательство. Функциональная эквивалентность $M_{3}^{\delta, \epsilon}$ и $M_{3}^{\alpha, \varphi}$ и выразимость базовых операций $M_{3}^{\alpha, \epsilon}$ и $M_{3}^{\delta, \varphi}$ :

- $x \supset^{\alpha} y=\neg^{\epsilon} \neg^{\epsilon}\left(x \supset^{\delta} y\right)$;

- $\neg^{\varphi} x=x \supset^{\delta} \neg^{\epsilon} x$;

- $x \supset^{\delta} y=\neg^{\varphi} \neg^{\varphi}\left(x \supset^{\alpha} y\right)$;

- $\neg^{\epsilon} x=x \supset^{\alpha} \neg^{\varphi} x$.

Теперь покажем, что через базовые операции $M_{3}^{\delta, \epsilon}$ нельзя выразить унарный оператор $f^{1}$, такой, что $f^{1}\left(\frac{1}{2}\right) \neq f^{1}(0)$, содержещий не меньше одного вхождения базовой операции.

Индуктивное допущение. Пусть $f^{1}$ нельзя выразить в $M_{3}^{\delta, \epsilon}$, используя менее $k(k \geq 1)$ вхождений $\supset^{\delta}$ и $\neg^{\epsilon}$.

Допустим, что $f^{1}$ можно выразить посредством суперпозиции $g$ операций $\supset^{\delta}$ и $\neg^{\epsilon}$, содержащей в точности $k$ вхождений данных операций.

Случай 1 . Пусть $g(x)=\neg^{\epsilon} h(x)$

1. $\neg^{\epsilon} h\left(\frac{1}{2}\right) \neq \neg^{\epsilon} h(0)$ (по условию)

2. $h(x)$ содержит $l(0<l<k)$ вхождений базовых операций или $h(x)$ есть $x$ (по условию)

3. Пусть $h(x)$ есть $x$ (допущение)

4. $\neg^{\epsilon} \frac{1}{2} \neq \neg^{\epsilon} 0$ (из 1,3 )

5. $\neg^{\epsilon} \frac{1}{2}=\neg^{\epsilon} 0$ (по определению $\neg^{\epsilon}$ )

6. $h(x)$ содержит $l$ вхождений базовых операций (из 2-5)

7. $h\left(\frac{1}{2}\right)=h(0)$ (из 6 по индуктивному допущению)

8. $\neg^{\epsilon} h\left(\frac{1}{2}\right)=\neg^{\epsilon} h(0)$ (из 7 по определению $\neg^{\epsilon}$ )

9. Неверно, что $g(x)=\neg^{\epsilon} h(x)$ (из 1,8$)$

Случай 2. Пусть $g(x)=h^{\prime}(x) \supset^{\delta} h^{\prime \prime}(x)$.

1. $h^{\prime}\left(\frac{1}{2}\right) \supset^{\delta} h^{\prime \prime}\left(\frac{1}{2}\right) \neq h^{\prime}(0) \supset^{\delta} h^{\prime \prime}(0)$ (по условию) 
2. $h^{\prime}(x)$ содержит $l(0<l<k)$ вхождений базовых операций или $h^{\prime}(x)$ есть $x$ (по условию)

3. $h^{\prime \prime}(x)$ содержит $m(0<m<k)$ вхождений базовых операций или $h^{\prime \prime}(x)$ есть $x$ (по условию)

4. Пусть $h^{\prime}(x)$ есть $x$ и $h^{\prime \prime}(x)$ есть $x$ (допущение)

5. $\frac{1}{2} \supset^{\delta} \frac{1}{2} \neq 0 \supset^{\delta} 0$ (из 1,4$)$

6. $\frac{1}{2} \supset^{\delta} \frac{1}{2}=0 \supset^{\delta} 0$ (по определению $\supset^{\delta}$ )

7. Неверно, что $h^{\prime}(x)$ есть $x$ и $h^{\prime \prime}(x)$ есть $x$ (из 5,6$)$

8. Пусть $h^{\prime}(x)$ содержит $l$ вхождений и $h^{\prime \prime}(x)$ содержит $m$ вхождений базовых операций.

9. $h^{\prime}\left(\frac{1}{2}\right)=h^{\prime}(0)$ (из 8 по индуктивному допущению)

10. $h^{\prime \prime}\left(\frac{1}{2}\right)=h^{\prime \prime}(0)$ (из 8 по индуктивному допущению)

11. $h^{\prime}\left(\frac{1}{2}\right) \supset^{\delta} h^{\prime \prime}\left(\frac{1}{2}\right)=h^{\prime}(0) \supset^{\delta} h^{\prime \prime}(0)$ (по определению $\supset^{\delta}$ )

12. Неверно, что $h^{\prime}(x)$ содержит $l$ вхождений и $h^{\prime \prime}(x)$ содержит $m$ вхождений базовых операций (из 1,11$)$.

13. Пусть $h^{\prime}(x)$ содержит $l$ вхождений базовых операций и $h^{\prime \prime}(x)$ есть $x$ (допущение)

14. $h^{\prime}\left(\frac{1}{2}\right) \supset^{\delta}\left(\frac{1}{2}\right) \neq h^{\prime}(0) \supset^{\delta}(0)$ (из 1,13$)$

15. $h^{\prime}\left(\frac{1}{2}\right) \supset^{\delta}\left(\frac{1}{2}\right)=\frac{1}{2}$ и $h^{\prime}(0) \supset^{\delta}(0)=1$, или $h^{\prime}\left(\frac{1}{2}\right) \supset^{\delta}\left(\frac{1}{2}\right)=1$ и $h^{\prime}(0) \supset^{\delta}(0)=\frac{1}{2}$ (из 14 по определению $\left.\supset^{\delta}\right)$

16. Пусть $h^{\prime}\left(\frac{1}{2}\right) \supset^{\delta}\left(\frac{1}{2}\right)=\frac{1}{2}$ и $h^{\prime}(0) \supset^{\delta}(0)=1$ (допущение)

17. $h^{\prime}\left(\frac{1}{2}\right)=1$ (из 16 по определению $\supset^{\delta}$ )

18. $h^{\prime}(0) \neq 1$ (из 16 по определению $\supset^{\delta}$ )

19. $h^{\prime}\left(\frac{1}{2}\right) \neq h^{\prime}(0)$ (из 17,18$)$

20. Неверно, что $h^{\prime}\left(\frac{1}{2}\right) \supset^{\delta}\left(\frac{1}{2}\right)=\frac{1}{2}$ и $h^{\prime}(0) \supset^{\delta}(0)=1$ (из 19 и индуктивного допущения) 
21. $h^{\prime}\left(\frac{1}{2}\right) \supset^{\delta}\left(\frac{1}{2}\right)=1$ и $h^{\prime}(0) \supset^{\delta}(0)=\frac{1}{2}$ (из 15,20$)$

22. $h^{\prime}\left(\frac{1}{2}\right) \neq 1$ (из 21 по определению $\supset^{\delta}$ )

23. $h^{\prime}(0)=1$ (из 21 по определению $\supset^{\delta}$ )

24. $h^{\prime}\left(\frac{1}{2}\right) \neq h^{\prime}(0) \quad$ из 22,23$)$

25. Неверно, что $h^{\prime}(x)$ содержит $l$ вхождений базовых операций и $h^{\prime \prime}(x)$ есть $x$ (из 24 и индуктивного допущения)

26. Пусть $h^{\prime}(x)$ есть $x$ и $h^{\prime \prime}(x)$ содержит $m$ вхождений базовых операций (допущение)

27. $\frac{1}{2} \supset^{\delta} h^{\prime \prime}\left(\frac{1}{2}\right)=\frac{1}{2}$ и $0 \supset^{\delta} h^{\prime \prime}(0)=1$, или $\frac{1}{2} \supset^{\delta} h^{\prime \prime}\left(\frac{1}{2}\right)=1$ и $0 \supset^{\delta} h^{\prime \prime}(0)=\frac{1}{2}$ (из 26 по определению $\supset^{\delta}$ ). Однако это невозможно в силу определения $\supset^{\delta}$.

28. Неверно, что $h^{\prime}(x)$ есть $x$ и $h^{\prime \prime}(x)$ содержит $m$ вхождений базовых операций (из 27)

29. Неверно, что $g(x)=h^{\prime}(x) \supset^{\delta} h^{\prime \prime}(x)$ (из $\left.2,3,4,12,25,28\right)$

Таким образом, через базовые операции $M_{3}^{\delta, \epsilon}$ нельзя выразить унарный оператор $f^{1}$, такой, что $f^{1}\left(\frac{1}{2}\right) \neq f^{1}(0)$, содержещий не меньше одного вхождения базовой операции.

Однако такой оператор выразим в $M_{3}^{\beta, \epsilon}, M_{3}^{\beta, \varphi}, M_{3}^{\gamma, \epsilon}$ и $M_{3}^{\gamma, \varphi}$ : $\left(x \supset^{\beta} x\right) \supset^{\beta} x,\left(x \supset^{\gamma} x\right) \supset^{\gamma} x . \quad$ Q.E.D.

УТВЕРЖДЕНИЕ 5. $M_{3}^{\gamma, \epsilon}$ функиионально эквивалентна $M_{3}^{\gamma, \varphi}$. В этих матрицах также выразимы базовые связки всех остальных матрии.

Доказательство. Чтобы доказать данное утверждение, достаточно следующих тождеств:

- $\neg^{\varphi} x=x \supset^{\gamma} \neg^{\epsilon} x$

- $\neg^{\epsilon} x=x \supset^{\gamma} \neg^{\varphi} x$

- $x \supset^{\beta} y=x \supset^{\gamma}\left(\neg^{\varphi} y \supset^{\gamma} y\right)$

- $x \supset^{\delta} y=\neg^{\varphi} \neg^{\varphi}\left(x \supset^{\gamma} y\right)$ 
Обобщая доказанные утверждения, можно заключить, что между матрицами $M_{3}^{\alpha, \epsilon}, M_{3}^{\beta, \epsilon}, M_{3}^{\gamma, \epsilon}, M_{3}^{\delta, \epsilon}, M_{3}^{\alpha, \varphi}, M_{3}^{\beta, \varphi}, M_{3}^{\gamma, \varphi}$, $M_{3}^{\delta, \varphi}$ имеет место порядок по отношению выразимости базовых связок. Причем, $M_{3}^{\gamma, \epsilon}$ и функционально эквивалентная ей $M_{3}^{\gamma, \varphi}$ выступают в роли максимума, $M_{3}^{\alpha, \epsilon}$ и $M_{3}^{\delta, \varphi}$ есть несравнимые минимумы, а $M_{3}^{\beta, \epsilon}, M_{3}^{\beta, \varphi}$ и $M_{3}^{\delta, \epsilon}$, функционально эквивалентная $M_{3}^{\alpha, \varphi}$ представляют собой три несравнимых промежуточных элемента.

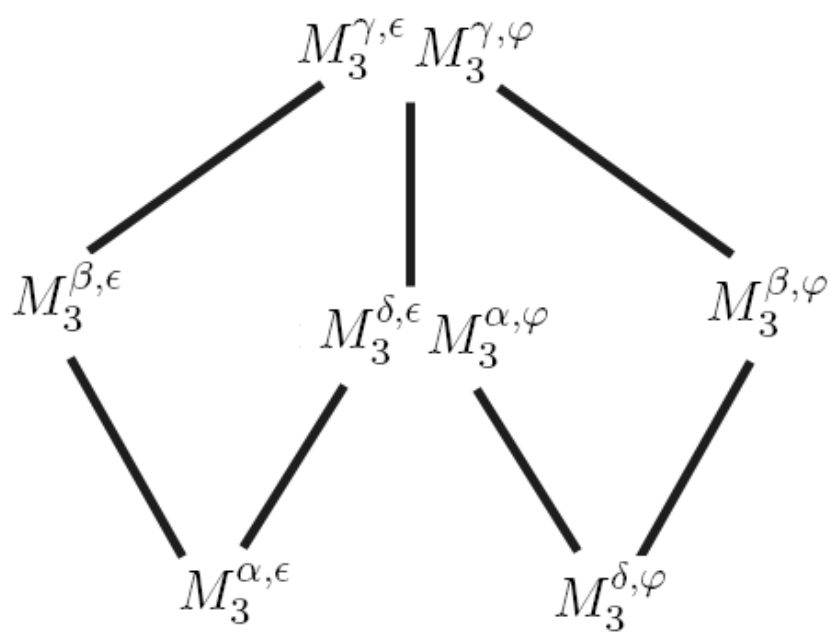

УТВЕРЖДДЕНИЕ 6. Набор базовых операчий $M_{3}^{\gamma, \epsilon}$ не является функционально полным в $P_{3}$.

Доказательство. Покажем, что через операции $\neg^{\epsilon}$ и $\supset^{\gamma}$ невыразим унарный оператор $f^{1}(x)$, такой что $f^{1}\left(\frac{1}{2}\right)=1$ и $f^{1}(0)=0$.

Индуктивное допущение. Пусть $f^{1}$ нельзя выразить в $M_{3}^{\gamma, \epsilon}$, используя менее $k$ вхождений $\supset^{\gamma}$ и $\neg^{\epsilon}$.

Теперь допустим, что $f^{1}$ можно выразить посредством суперпозиции $g$ операций $\supset^{\gamma}$ и $\neg^{\epsilon}$, содержащей в точности $k$ вхождений данных операций.

Случай 1. Пусть $g(x)$ есть $\neg^{\epsilon} h(x)$

1. $\neg^{\epsilon} h\left(\frac{1}{2}\right)=1$ и $\neg^{\epsilon} h(0)=0$ (по условию) 
2. $h\left(\frac{1}{2}\right) \neq 1$ и $h(0)=1$ (в силу определения $\neg^{\epsilon}$ )

3. $h(x)$ содержит по меньшей мере одну операцию и имеет вид $\neg^{\epsilon} h^{*}(x)$ или $h^{\prime}(x) \supset^{\gamma} h^{\prime \prime}(x)$ (из 2)

4. Пусть $h(x)$ имеет вид $\neg^{\epsilon} h^{*}(x)$ (допущение)

5. $\neg^{\epsilon} h^{*}\left(\frac{1}{2}\right) \neq 1$ и $\neg^{\epsilon} h^{*}(0)=1$ (из 2 и 4 )

6. $h^{*}\left(\frac{1}{2}\right)=1$ и $h^{*}(0)=0$ (из 5 по определеню $\neg^{\epsilon}$ ). Однако это противоречит индуктивному допущению. Следовательно, неверно, что $h(x)$ имеет вид $\neg^{\epsilon} h^{*}(x)$.

7. $h(x)$ имеет вид $h^{\prime}(x) \supset^{\gamma} h^{\prime \prime}(x)$ (из 3 и 6 )

8. $h^{\prime}\left(\frac{1}{2}\right) \supset^{\gamma} h^{\prime \prime}\left(\frac{1}{2}\right) \neq 1$ и $h^{\prime}(0) \supset^{\gamma} h^{\prime \prime}(0)=1$ (из 1,7$)$

9. $h^{\prime}\left(\frac{1}{2}\right)=1$ и $h^{\prime \prime}\left(\frac{1}{2}\right) \neq 1$ (из 8)

10. $h^{\prime}(0) \neq 1$ или $h^{\prime \prime}(0)=1$ (из 8$)$

11. Пусть $h^{\prime}(0) \neq 1$ (Допущение)

12. $\neg^{\epsilon} \neg^{\epsilon} h^{\prime}(0)=0$ (из 11 по определению $\neg^{\epsilon}$ )

13. $\neg^{\epsilon} \neg^{\epsilon} h^{\prime}\left(\frac{1}{2}\right)=1$ (из 9 по определению $\left.\neg^{\epsilon}\right)$

14. $\neg^{\epsilon} \neg^{\epsilon} h^{\prime}(x)$ содержит менее $k$ вхождений связок. Следовательно, неверно, что $h^{\prime}(0) \neq 1$ (из 12,13 и индуктивного допущения)

15. $h^{\prime \prime}(0)=1($ из 10,14$)$

16. $\neg^{\epsilon} h^{\prime \prime}(0)=0$ (из 15 по определению $\neg^{\epsilon}$ )

17. $\neg^{\epsilon} h^{\prime \prime}\left(\frac{1}{2}\right)=1$ (из 9 по определению $\neg^{\epsilon}$ )

18. $\neg^{\epsilon} h^{\prime \prime}(x)$ содержит менее $k$ вхождений связок. Неверно, что $g(x)$ есть $\neg^{\epsilon} h(x)$ (из 16,17 и индуктивного допущения)

Случай 2. Пусть $g(x)$ есть $h^{\prime}(x) \supset^{\gamma} h^{\prime \prime}(x)$

1. $h^{\prime}\left(\frac{1}{2}\right) \supset^{\gamma} h^{\prime \prime}\left(\frac{1}{2}\right)=1$ и $h^{\prime}(0) \supset^{\gamma} h^{\prime \prime}(0)=0$ (по условию)

2. $h^{\prime}(0)=1$ и $h^{\prime \prime}(0)=\frac{1}{2}$ (из 1 по определению $\supset^{\gamma}$ ) 
3. $h^{\prime}\left(\frac{1}{2}\right) \neq 1$ или $h^{\prime \prime}\left(\frac{1}{2}\right)=1$ (из 1 по определению $\supset^{\gamma}$ )

4. Пусть $h^{\prime}\left(\frac{1}{2}\right) \neq 1$ (Допущение)

5. $\neg^{\epsilon} h^{\prime}\left(\frac{1}{2}\right)=1$ (из 4 по определению $\neg^{\epsilon}$ )

6. $\neg^{\epsilon} h^{\prime}(0)=0$ (из 2 по определению $\neg^{\epsilon}$ )

7. $\neg^{\epsilon} h^{\prime}(x)$ содержит менее $k$ вхождений операций. Таким образом, неверно, что $h^{\prime}\left(\frac{1}{2}\right) \neq 1$ (из 5,6 и индуктивного допущения)

8. $h^{\prime \prime}\left(\frac{1}{2}\right)=1$ (из 3,7$)$

9. $\neg^{\epsilon} \neg^{\epsilon} h^{\prime \prime}\left(\frac{1}{2}\right)=1$ (из 8 по определению $\neg^{\epsilon}$ )

10. $\neg^{\epsilon} \neg^{\epsilon} h^{\prime \prime}(0)=0$ (из 2 по определению $\neg^{\epsilon}$ )

11. $\neg^{\epsilon} \neg^{\epsilon} h^{\prime \prime}(x)$ содержит $m(m \leq k)$ операций. Однако это противоречит индуктивному допущению и Случаю 1 настоящего доказательства.

Таким образом, набор базовых операций $M_{3}^{\gamma, \epsilon}$ не является функционально полным в $P_{3}$.

Q.E.D.

Мы показали, что различные трехэлементные матрицы с классическим отношением логического следования не только различаются по силе с функциональной точки зрения, но и образуют достаточно интересную структуру. Можно сделать вывод, что функциональная полнота системы базовых связок в импликативно-негативной логической матрице не является необходимым свойством классической логики высказываний.

В стандартной двузначной матрице классические конъюнкция и дизъюнкция обладают совйствами решеточных операторов. То есть они удовлетворяют следующим тождествам:

- Идемпотентность: $x \vee x=x, x \wedge x=x$;

- Коммутативность: $x \vee y=y \vee x, x \wedge y=y \wedge x$;

- Ассоциативность: $x \vee(y \vee z)=(x \vee y) \vee z, x \wedge(y \wedge z)=$ $(x \wedge y) \wedge z$ 
- Поглощение: $x \vee(x \wedge y)=x, x \wedge(x \vee y)=x$.

Так ли это для рассмотренных выше матриц? Выразим дизъюнкцию через импликацию и отрицание:

$$
x \vee y=\neg x \supset y .
$$

В зависимости от выбранных $\neg$ и $\supset$ может получиться одна из следующих связок.

\begin{tabular}{|c|ccc|}
\hline$\vee^{1}$ & 1 & $\frac{1}{2}$ & 0 \\
\hline 1 & 1 & 1 & 1 \\
$\frac{1}{2}$ & 1 & 0 & 0 \\
0 & 1 & 0 & 0 \\
\hline
\end{tabular}

\begin{tabular}{|c|ccc|}
\hline$\bigvee^{2}$ & 1 & $\frac{1}{2}$ & 0 \\
\hline 1 & 1 & 1 & 1 \\
$\frac{1}{2}$ & 1 & $\frac{1}{2}$ & 0 \\
0 & 1 & $\frac{1}{2}$ & 0 \\
\hline
\end{tabular}

\begin{tabular}{|c|ccc|}
\hline$\vee^{3}$ & 1 & $\frac{1}{2}$ & 0 \\
\hline 1 & 1 & 1 & 1 \\
$\frac{1}{2}$ & 1 & 0 & $\frac{1}{2}$ \\
0 & 1 & 0 & $\frac{1}{2}$ \\
\hline
\end{tabular}

\begin{tabular}{|c|ccc|}
\hline$\bigvee^{4}$ & 1 & $\frac{1}{2}$ & 0 \\
\hline 1 & 1 & 1 & 1 \\
$\frac{1}{2}$ & 1 & $\frac{1}{2}$ & $\frac{1}{2}$ \\
0 & 1 & $\frac{1}{2}$ & $\frac{1}{2}$ \\
\hline
\end{tabular}

Операции $\vee^{1}$ сответствуют $M_{3}^{\alpha, \epsilon}$ и $M_{3}^{\alpha, \varphi}$. Операции $\vee^{2}$ сответствуют $M_{3}^{\beta, \epsilon}$ и $M_{3}^{\beta, \varphi}$. Операции $\vee^{3}$ сответствуют $M_{3}^{\gamma, \epsilon}$ и $M_{3}^{\gamma, \varphi}$. Операции $\vee^{4}$ сответствуют $M_{3}^{\delta, \epsilon}$ и $M_{3}^{\delta, \varphi}$.

Теперь определим конъюнкцию через ипликацию и отрицание:

$$
x \wedge y=\neg(x \supset \neg y) .
$$

В зависимости от выбранных $\neg$ и $\supset$ возможны два варианта.

\begin{tabular}{|c|ccc|}
\hline$\wedge^{1}$ & 1 & $\frac{1}{2}$ & 0 \\
\hline 1 & 1 & 0 & 0 \\
$\frac{1}{2}$ & 0 & 0 & 0 \\
0 & 0 & 0 & 0 \\
\hline
\end{tabular}

\begin{tabular}{|c|ccc|}
\hline$\wedge^{2}$ & 1 & $\frac{1}{2}$ & 0 \\
\hline 1 & 1 & $\frac{1}{2}$ & $\frac{1}{2}$ \\
$\frac{1}{2}$ & $\frac{1}{2}$ & $\frac{1}{2}$ & $\frac{1}{2}$ \\
0 & $\frac{1}{2}$ & $\frac{1}{2}$ & $\frac{1}{2}$ \\
\hline
\end{tabular}

Операции $\wedge^{1}$ соответствуют $M_{3}^{\alpha, \epsilon}, M_{3}^{\beta, \epsilon}, M_{3}^{\gamma, \epsilon}, M_{3}^{\delta, \epsilon}$. Операции $\wedge^{2}$ соответствуют $M_{3}^{\alpha, \varphi}, M_{3}^{\beta, \varphi}, M_{3}^{\gamma, \varphi}, M_{3}^{\delta, \varphi}$. 


\begin{tabular}{|c|c|c|c|c|}
\hline & $M_{3}^{\alpha, \epsilon}$ & $M_{3}^{\beta, \epsilon}$ & $M_{3}^{\gamma, \epsilon}$ & $M_{3}^{\delta, \epsilon}$ \\
\hline$x \vee x=x$ & - & + & - & - \\
\hline$x \wedge x=x$ & - & - & - & - \\
\hline$x \vee y=y \vee x$ & + & - & - & + \\
\hline$x \wedge y=y \wedge x$ & + & + & + & + \\
\hline$x \vee(y \vee z)=(x \vee y) \vee z$ & + & + & - & + \\
\hline$x \wedge(y \wedge z)=(x \wedge y) \wedge z$ & + & + & + & + \\
\hline$x \vee(x \wedge y)=x$ & - & - & - & - \\
\hline$x \wedge(x \vee y)=x$ & - & - & - & - \\
\hline
\end{tabular}

\begin{tabular}{|c|c|c|c|c|}
\hline & $M_{3}^{\alpha, \varphi}$ & $M_{3}^{\beta, \varphi}$ & $M_{3}^{\gamma, \varphi}$ & $M_{3}^{\delta, \varphi}$ \\
\hline$x \vee x=x$ & - & + & - & - \\
\hline$x \wedge x=x$ & - & - & - & - \\
\hline$x \vee y=y \vee x$ & + & - & - & + \\
\hline$x \wedge y=y \wedge x$ & + & + & + & + \\
\hline$x \vee(y \vee z)=(x \vee y) \vee z$ & + & + & - & + \\
\hline$x \wedge(y \wedge z)=(x \wedge y) \wedge z$ & + & + & + & + \\
\hline$x \vee(x \wedge y)=x$ & - & - & - & - \\
\hline$x \wedge(x \vee y)=x$ & - & - & - & - \\
\hline
\end{tabular}

Таким образом, ни в одной из рассматриваемых матриц дизъюнкция и конъюнкция не обладают свойствами решеточных операторов.

\section{Литература}

[1] Бочвар Д.А. Об одном трехзначном исчислении и его применении к анализу парадоксов классического расширенного функционального исчисления // Математический сборник. Т. 4, № 2. 1938. С. 287-308.

[2] Девяткин Л.Ю. Многозначные изоморфы классической пропозициональной логики. Кандидатская диссертация на соискание ученой степени кандидата философских наук. М., 2008. С. 29.

[3] Менделъсон Э. Введение в математическую логику. М., 1984. С. 33.

[4] Avron A. Natural 3-valued logics - characterization and proof theory // The Journal of Symbolic Logic. Vol. 56. № 1. P. 276-294.

[5] Monteiro A. Construction des algebres de Lukasiewicz trivalentes dans les algebres de Boole monadiques, I // Mathematica Japonica. Vol. 12. P. 1-23.

[6] Stupecki J., Bryl J. and Prucnal T. Some remarks on the three-valued logic of J. Jukasiewicz // Studia Logica. Vol. 21. P. 45-70. 